\title{
Cognitive Control: Dynamic, Sustained, and Voluntary Influences
}

\author{
Diego Fernandez-Duque and MaryBeth Knight \\ Villanova University
}

\begin{abstract}
The cost of incongruent stimuli is reduced when conflict is expected. This series of experiments tested whether this improved performance is due to repetition priming or to enhanced cognitive control. Using a paradigm in which Word and Number Stroop alternated every trial, Experiment 1 assessed dynamic trial-to-trial changes. Incongruent trials led to task-specific reduction of conflict (trial $n+2$ ) without cross-task modulation (trial $n+1$ ), but this was fully explained by repetition priming. In contrast, an increased ratio of incongruent words did lead to sustained task-specific enhancement, above and beyond repetition priming (Experiment 2). Experiments 3 and 4 assessed the voluntary modulation of cognitive control: A cue predicted the congruency of the incoming trial, allowing participants to establish the correct mindset (Word Stroop in Experiment 3, Flanker task in Experiment 4). Preparing oneself to process an incongruent word (or flanker) enhanced conflict resolution in the subsequent Number Stroop, an example of cross-task modulation. Taken together, these experiments reveal the multifaceted aspects of conflict resolution: Trial-to-trial changes are often due to repetition priming; sustained modulations brought about by task demands are task specific; and voluntary modulations are task general.
\end{abstract}

Keywords: executive function, attention, switching, modularity

The term cognitive control refers to the ability to establish a mindset that overcomes the automatic response in favor of a less salient or more novel answer (Logan, 1985; Posner \& DiGirolamo, 1998; Posner \& Fernandez-Duque, 1999). In a classic example of cognitive control, participants are asked to name the color of ink in which a color word is displayed (Stroop, 1935). When there is a mismatch of word and ink, cognitive control is required to suppress the impulse of reading the word, a process that requires effort and is error prone. The difference in performance between "incongruent trials" and trials in which word and ink match ("congruent") is a measure of cognitive control called the congruency effect.

The congruency effect is seldom eliminated but can be reduced by many factors, such as (a) the presence of an incongruent stimulus in the preceding trial, (b) the increase of incongruent-tocongruent ratio, and (c) the presence of a cue warning about trial type (Botvinick, Nystrom, Fissell, Carter, \& Cohen, 1999; Gratton, Coles, \& Donchin, 1992; Logan \& Zbrodoff, 1979; MacDonald, Cohen, Stenger, \& Carter, 2000; Melara \& Algom, 2003; Swick \& Jovanovic, 2002; Tzelgov, Henik, \& Berger, 1992). ${ }^{1}$ Although these findings are uncontroversial, the mechanisms by which they are brought about and their theoretical significance are a matter of great debate.

Some researchers have claimed that changes in congruency effect are truly caused by fluctuations in cognitive control (Botvin-

Diego Fernandez-Duque and MaryBeth Knight, Psychology Department, Villanova University.

Several Villanova University students helped collect data for this study, including Patrick Crandell, Adrienne Fallon, Sarah Irvin, Jessica Landers, and Adriana Lombardi. We thank Ulrich Mayr and Mike Wendt for helpful criticism, and Mike Wendt for suggesting Experiment 4.

Correspondence concerning this article should be addressed to Diego Fernandez-Duque, Psychology Department, Villanova University, 800 Lancaster Ave, Villanova, PA 19085. E-mail: diego.fernandezduque@villanova.edu ick, Braver, Barch, Carter, \& Cohen, 2001; Kunde \& Wühr, 2006 Notebaert, Gevers, Verbruggen, \& Liefooghe, 2006; Ridderinkhof, Ullsperger, Crone, \& Nieuwenhuis, 2004; Stürmer, Leuthold, Soetens, Schröter, \& Sommer, 2002; Swick \& Jovanovic, 2002), while others argue that the reduction in congruency effect is due to stimulus repetition priming. According to the former view, interference is reduced following an incongruent trial because the effort of resolving conflict in trial $n$ leads participants to adjust their attentional control in future trials. Incongruent trials act as reminders that help participants to maintain a task mindset (e.g., focus on the ink and ignore the word). For example, the conflict monitoring theory argues that conflict is first detected by midfrontal regions of the brain (conflict monitoring stage) and later relayed to dorsolateral prefrontal areas acting at the cognitive control stage; this prepares the system to more effectively filter incongruent information (Botvinick et al., 2001). In these models, the detection of conflict is causally effective for the production of cognitive control.

Other research, however, has questioned the use of congruency effect as a proxy for cognitive control, arguing instead that changes in congruency effects can be accounted for by stimulus repetition priming (Logan \& Bundesen, 2003; Mayr, Awh, \& Laurey, 2003; Notebaert, Verbruggen, \& Soetens, 2005). According to this view, the reduction in congruency effect after an incongruent trial is a marker of decreased task demand, not a marker of increased cognitive control. Participants respond quickly after incongruent trials not because cognitive control is heightened but because the correct response is salient and bypasses conflict altogether. This argument is based on the fact that stimulus repetition trials by

\footnotetext{
${ }^{1}$ This is not meant as an exhaustive list, as many other factors have been shown to modulate the congruency effect, including practice (Reisberg, Baron, \& Kemler, 1980), hypnotic suggestibility (Raz, Shapiro, Fan, \& Posner, 2002), input-output agreement (Durgin, 2000), and developmental differences (Rueda et al., 2004).
} 
definition have the same congruency as their preceding trial- both are congruent or both are incongruent. Therefore the congruency effect-the reaction time (RT) difference between incongruent and congruent trials-is reduced after an incongruent trial because some incongruent trials are primed but none of the congruent trials are. The same logic is used to explain the increase in congruency effect following a congruent trial (some of the congruent trials will be primed but none of the incongruent trials will).

Although some studies have provided support for the stimulus repetition priming hypothesis, others have not (Kerns et al., 2004; Kunde \& Wühr, 2006; Notebaert et al., 2006; Ullsperger, Bylsma, $\&$ Botvinick, 2005). Furthermore, the debate has centered on trial-to-trial fluctuations in congruency effect; no studies have yet explored whether sustained effects that occur over the course of a block of trials can also be accounted for by repetition priming. Finally, repetition priming does not explain the willful modulation of congruency effects, as when a participant is told to set his or her mind for a difficult task (Gratton et al., 1992). Thus, repetition priming may account for some effects previously attributed to cognitive control but not others. An important goal of our study was to investigate the conditions under which repetition priming may account for such modulations.

A theoretically more ambitious goal was to explore the task specificity of cognitive control modulation. Does experiencing conflict in one task prepare one to better filter the irrelevant information in another task, or is the benefit highly specific, thus generalizing only to same-task trials? Turning the question around, one can ask if it is possible to set separate levels of cognitive control for different concurring tasks, or is one bound to use the same criterion for both tasks? An answer to these questions would shed light on whether cognitive control is subserved by a single supervisory mechanism, a question that has been the focus of much interest in recent years (Corballis \& Gratton, 2003; Miyake, Friedman, Emerson, Witzki, \& Howerter, 2000).

The conflict monitoring theory provides an excellent place from which to address such a question of task specificity. The theory has been very influential not only because of its account of empirical data, but also because its clear tenets and explicit assumptions have led to several testable predictions (Botvinick et al., 2001). A main claim of the theory is the anatomical overlap of the conflict monitoring system; error detection, perceived uncertainty, and response conflict are all said to tap into the same brain system. Although some anatomical segregation may exist based on input type (error, uncertainty, conflict), there seems to exist a common output (perceived difficulty). A second important claim is the high level of abstraction at which information is processed-very different physical input may affect the monitoring system. Third, no distinctions are made based on the timing or triggering factors of modulation. Thus, trial-to-trial fluctuations and sustained changes are considered to be two instantiations of the same system, and whether the modulation is brought about by task demands or by the participant's will is not explicitly addressed in the theoretical models. It follows from these claims that the theory posits the existence of a very general system for monitoring conflict. Does the output of this system feed into an equally generic system for cognitive control? In other words, is there a task-general stage in information processing, at which a single supervisory system applies top-down cognitive control broadly, in response to perceived conflict? Or, instead, is top-down control always task-specific, and therefore it is impossible to establish a mindset for processing conflict in an abstract sense? This latter view would be consistent with theories postulating that different stimulus dimensions are activated specifically based on their informational value (Melara \& Algom, 2003). To shed light on this question, the current study assessed whether a single level of control would be set for two alternating streams of conflict tasks. The presence of carry-over effects across tasks would generalize to the stage of cognitive control the generic system posed by the conflict monitoring theory for the conflict detection stage. Carry-over effects would also provide strong evidence against the stimulus repetition priming hypothesis.

Most of our experiments made use of two versions of the Stroop task (word, number). In the classic version of this paradigm, participants are asked to name the color of ink in which a word is presented (Stroop, 1935). The word itself is a color name that is either different from the color of ink used to present it (incongruent) or is the same as the ink color (congruent). In the Number Stroop task, participants are asked to name the number of digits displayed in a string of identical numbers, instead of giving the more automatic response of the digit's identity. The quantity and digit identity can either be congruent $(22 ; 1)$ or incongruent $(33$; 222).

In both of these versions, the conflict posed by incongruent trials leads to slower reaction times, increased number of errors, and increased activation of mediofrontal and dorsolateral prefrontal cortex areas (Bush, Luu, \& Posner, 2000; Bush et al., 1998; MacLeod, 1991; Ridderinkhof, Ullsperger, Crone, \& Nieuwenhuis, 2004). These areas correspond to those described by the conflict monitoring theory as important for conflict monitoring and cognitive control. The behavioral and neuroimaging similarity between the two tasks is consistent with the existence of a generic (i.e., non-task-specific) control system. It predicts that increased task demands in one task (e.g., the presence of incongruent trials) will trigger increased cognitive control in the other task. Experiments 1 and 2 tested this hypothesis.

Cognitive control can be increased exogenously by changes in task demands but can also be increased endogenously by participants' voluntary intention, conceivable with different patterns of cross-task modulation. Prefrontal areas involved in conflict resolution are activated and congruency effect is reduced when participants are warned about the imminent occurrence of incongruent information (Gratton et al., 1992; MacDonald et al., 2000). However, although the literature supports the claim that participants can voluntarily set their minds to resolve conflict in a specific task, less is known about whether the benefit of endogenous modulation generalizes across tasks. We tested the cross-task effect of voluntary cues in Experiments 3 and 4.

In sum, a reduction in the congruency effect can be brought about by (a) the presence of an incongruent stimulus in the previous trial, (b) the increase of the incongruent-to-congruent ratio, and (c) the presence of a cue predicting the next target type. The mechanism invoked to reduce congruency effect may have a major role in determining whether repetition priming is at play and whether the modulation is task specific or task general. In other words, whether a single or multiple system is at work and to what degree repetition priming is exerting an influence might depend on the properties of the task. For this reason, separate experiments were run to assess all three forms of congruency effect modulation. 
Experiment 1 explored the phasic trial-to-trial modulation of cognitive control. Experiment 2 looked at more sustained modulations brought about by changes in the task demands across blocks of trials (i.e., varying the ratio of incongruent to congruent trials). Experiments 3 and 4 assessed the endogenous modulation of cognitive control in a voluntary cueing task.

\section{Experiment 1}

Experiment 1 presented alternating trials of Word and Number Stroop tasks to investigate trial-to-trial changes in cognitive control. We asked whether congruency effect following an incongruent trial would be reduced across tasks (trial $n+1$ ), within task (trial $n+2$ ), or both. For example, we asked whether an incongruent word in trial $n$ would reduce conflict in trial $n+1$ (Number Stroop), in trial $n+2$ (Word Stroop), or both. As a means of maximizing the chances of finding cross-task modulation, the same response modality was used across tasks. To the extent that conflict is resolved at least in part at the level of response selection, response overlap should maximize the chances of finding crosstask modulation.

Cross-task modulation could not be explained by stimulus priming because stimuli are different across tasks. In contrast, withintask modulation may be explained by stimulus priming. Thus, we also assessed stimulus priming as a possible mechanism of withintask modulation.

\section{Method}

Participants. For this and all other experiments, participants were undergraduate students enrolled in an introductory psychology course who completed the task for course credit and reported normal or corrected-to-normal visual acuity and color vision. Forty-two students (30 women; mean age $=19.1$ years, $S D=1.2$ years, range $=18-23$ years) participated in Experiment 1 . All but 1 participant were right-handed.

Apparatus and stimuli. For the Word Stroop task, nine stimuli were created by the combination of three words (red, blue, green) and font colors (red, blue, green). For the Number Stroop, three digits $(1,2,3)$ were displayed in three different set sizes $(1,2,3)$ in a white font. Stimuli were displayed centrally in 36-point Courier New font on a black background. For this and all other experiments, stimuli were displayed on a 17 -inch monitor set to a screen resolution of $1,024 \times 768$ pixels. Data were collected via the keyboard of a Dell computer equipped with a Pentium 4 processor and Windows XP. The timing of the stimulus display and data collection were managed using E-prime, a commercial experiment application.

Procedure. Stimulus alternated between Word and Number task on every trial (see Figure 1). For each task, there were 72 congruent trials (e.g., 333 for the Number task) and 72 incongruent trials (e.g., 33). Half of these trials were preceded by a congruent trial of the other task (e.g., word red in red ink), and the other half were preceded by an incongruent trial (e.g., word red in green ink). Thus, there were 36 trials in each cell of interest, for a total of 288 trials in a single block lasting approximately $15 \mathrm{~min}$. Trials occurred in a continuous sequence.

In each trial, the target remained on display until response or for a maximum of $5 \mathrm{~s}$ and was followed by a 500-ms response-

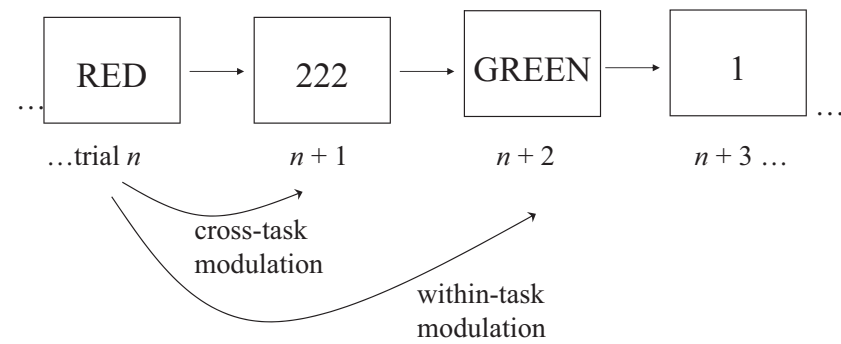

Figure 1. Design of Experiment 1. The tasks alternated every trial between Word and Number Stroop. Each trial modulated subsequent trials and was influenced by previous trials. The modulation could be across tasks (the effect of trial $n$ on trial $n+1$ ) or within task (the effect of trial $n$ on trial $n+2$ ). Illustrated here is a case in which the modulator task (trial $n$ ) was Word Stroop. For the Word Stroop, the ink color was congruent or incongruent with the word (not shown in the figure).

stimulus interval. Participants responded with their right hands by pressing the keys $J, K$, or $L$. The same keys were used in both tasks, and they indicated, respectively, red, blue, and green, and set size of one, two, and three. The stimulus-response mapping was the same for all participants. After a practice session (15-20 trials), which the researcher observed to ensure participant understanding, the researcher left the participant alone in a private room to complete the experiment and instructed the participant to work as quickly as possible while minimizing errors.

\section{Results}

Incorrect responses $(4.3 \%$ of Word trials, $3.4 \%$ of Number trials) and the two trials that immediately followed errors were excluded from the RT analyses. We also excluded the first two trials of the block, trials with no response, anticipatory responses ( RT $<200 \mathrm{~ms}$ ), and unusually slow responses (RT $>2,500 \mathrm{~ms}$ for the Word task; RT $>1,500$ for the Number task). A total of $1 \%$ of trials were excluded for these reasons.

Each trial served a dual purpose of modulator and probe. In other words, trial $n$ could modulate trials $n+1$ (across tasks) and $n+2$ (within task), and at the same time performance in trial $n$ would be influenced by preceding trials $n-1$ and $n-2$. We labeled trial $n$ to be the modulator trial and trials $n+1$ and $n+$ 2 to be the probe trials. Error rates and mean RTs were calculated for the modulator task (Word, Number), probe task (Word, Number), modulator trial type (congruent, incongruent), and probe trial type (congruent, incongruent). Cross-task modulations were assessed by cells with different modulator and probe tasks (trial $n$, trial $n+1)$. Within-task modulations were assessed by cells with same modulator and probe tasks (trial $n$, trial $n+2$ ).

Absence of cross-task trial-to-trial modulation. Would the presence of conflict in the Word Stroop improve the ability to resolve conflict in the immediately following Number Stroop? As a means of answering this question, data from the number task were submitted to a within-subject analysis of variance (ANOVA) with two variables: Word trial type (congruent, incongruent) and Number trial type (congruent, incongruent). As expected, responses to incongruent Number trials were slower than responses to congruent trials $\left(M_{\text {incg }}=730 \mathrm{~ms} ; M_{c g}=645 \mathrm{~ms}\right.$ [incg $=$ incongruent and $\mathrm{cg}=$ congruent $]), F(1,41)=279, M S E=1,090$, 
$p<.001$. A response to a number following an incongruent word was slower than a response to a number following a congruent word by a small $(20 \mathrm{~ms})$ but statistically significant amount, $F(1$, $41)=17.9, \operatorname{MSE}=721, p<.001$. More important, an incongruent Word trial failed to modulate the congruency effect in the following Number trial (congruency effect following an incongruent word: $89 \mathrm{~ms}$; congruency effect following a congruent word: $82), F(1,41)=0.8, M S E=696, n s$ (see Figure 2A and Table 1). The error data similarly revealed that congruency effect in the Number Stroop, $F(1,41)=79, M S E=12.3, p<.001$, was not modulated by the congruency of the preceding Word trial, $F(1$, $41)=0.5, M S E=5.4, n s$ (see Table 2). Although the experiment was designed to assess the effect of the Word Stroop on the Number Stroop, one could also assess the effect of the Number Stroop on the Word Stroop. This supplementary analysis also failed to reveal cross-task modulation (149 ms vs. $151 \mathrm{~ms}), F(1$, 41) $=0.2, \operatorname{MSE}=1,326, \mathrm{~ns}$.

Presence of within-task trial-to-trial modulation. Would the presence of conflict in the Word Stroop improve the ability to resolve conflict in the next Word Stroop trial (i.e., $n+2$ trial)? As a means of assessing this within-task modulation, data were submitted to a within-subject ANOVA with two variables: Word trial type (congruent, incongruent) and $n+2$ trial type (congruent, incongruent). Unlike the cross-task data, incongruent words did reduce the cost of conflict resolution for the next Word trial (Congruency effect following an incongruent word: $132 \mathrm{~ms}$; congruency effect following a congruent word: $168 \mathrm{~ms}$ ), $F(1,41)=$ 6.7, $M S E=2,117, p<.01$ (see Figure 2B). The same pattern was found for the accuracy data, $F(1,41)=5.4, M S E=4.7, p<.02$. Although the experiment was designed to assess the within-task effect of the Word Stroop, it is also possible to explore the possible within-task modulation of the Number Stroop. Such analysis revealed no evidence of within-task modulation for the Number task (87 ms vs. $84 \mathrm{~ms}), F(1,41)=0.1, M S E=848, n s$.

Priming contributes to within-task trial-to-trial modulation. To control for priming effects, we redid the within-task analysis after filtering those trials in which the word-ink combination was an exact replica of the preceding trial. In the absence of stimulus repetition priming, the congruency effect, $F(1,41)=170.0, p<$ .001 , was not modulated by the type of trial preceding it (congruency effect following an incongruent word: $150 \mathrm{~ms}$; congruency effect following a congruent word: $133 \mathrm{~ms}), F(1,41)=1.2$, $M S E=2,648, n s$ (see Figure 2C). The error data also failed to reveal a modulation, $F(1,41)=1.2, M S E=4.7$, $n s$.

To further explore the existence of priming, we contrasted repetition and nonrepetition trials. This was a subset of trials $n+$ 2 of the Word task. To control for the fact that repetition trials are always preceded by the same kind of congruency - that is, congruent trials are preceded by congruent ones, and incongruent trials are preceded by incongruent ones-we limited the analysis of nonrepetition trials to trials that were preceded by the same kind of congruency. An analysis including repetition priming (yes, no) and congruency (congruent, incongruent) revealed a main effect of congruency, $F(1,41)=98.0, M S E=9,395, p<.001$, and, more important, a main effect of priming, $F(1,41)=40.0, M S E=$ $11,506, p<.001\left(M_{\text {Prime }}=815 \mathrm{~ms} ; M_{\text {NoPrime }}=920 \mathrm{~ms}\right)$. Taken together, these findings argue for the existence of repetition priming that accounts for the reduction in congruency effect following within-task incongruent information.

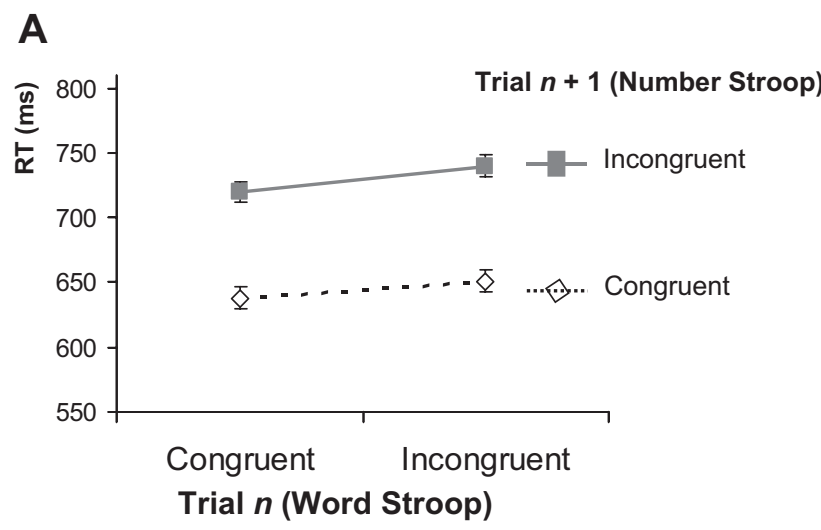

B
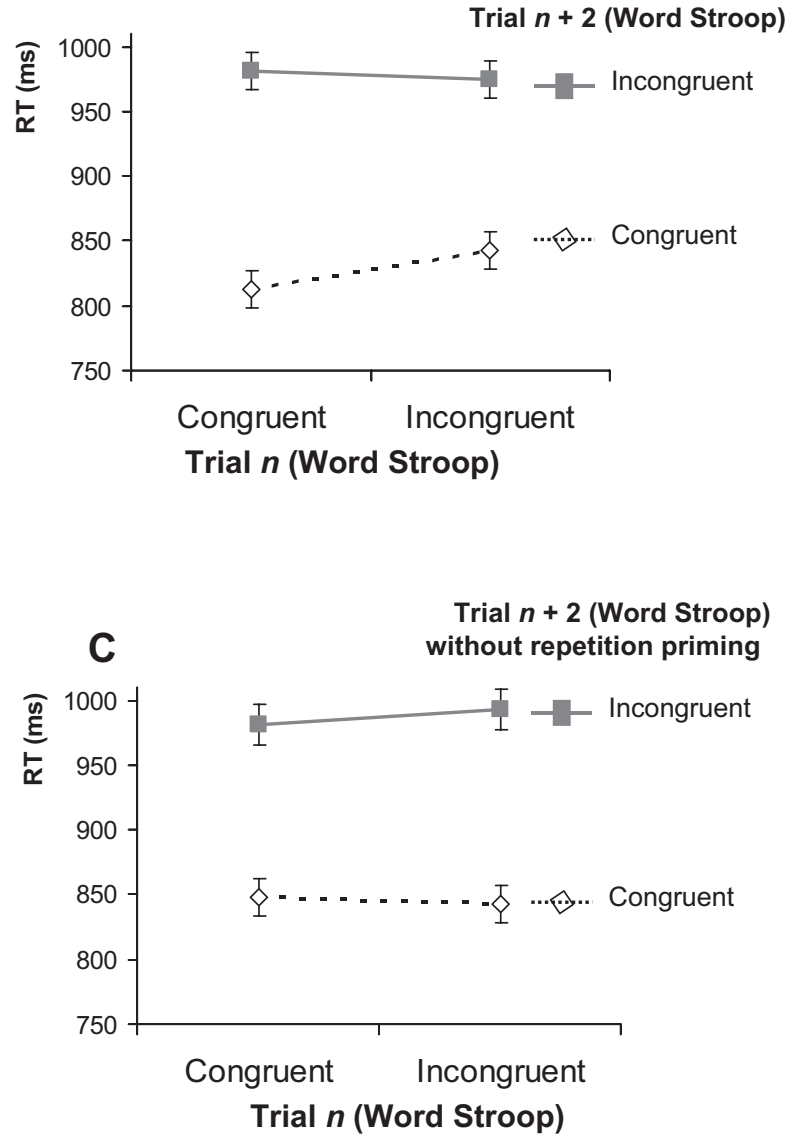

Figure 2. A-C: Dynamic, trial-to-trial changes in congruency effect in Experiment 1 as revealed by reaction times. Error bars show 95\% confidence intervals based on the mean square error of the interaction, to partial out between-subjects variance (following Loftus \& Masson, 1994). A: Absence of cross-task modulation. The congruency effect in the Number Stroop (trial $n+1$ ) was unaffected by the Word Stroop trial preceding it (congruent, incongruent). B: Presence of within-task modulation. The congruency effect in the Word Stroop (trial $n+2$ ) was reduced by the occurrence of an incongruent Word Stroop trial. C: Absence of within-task modulation in the absence of repetition priming. The filtering out of trials in which the word-ink display repeated from the preceding trial was enough to get rid of the within-task modulation. $\mathrm{RT}=$ reaction time. 
Table 1

Experiment 1 Reaction Times (RT) Showing Cross- and Within-Task Trial-to-Trial Modulation Caused by the Word Stroop

\begin{tabular}{|c|c|c|c|c|c|c|}
\hline \multirow[b]{3}{*}{ Influence of Word Stroop (trial $n$ ) } & \multicolumn{4}{|c|}{ Probe trial } & \multirow{3}{*}{$\begin{array}{l}\text { Congruency } \\
\text { effect }\end{array}$} & \multirow[b]{3}{*}{ Modulation } \\
\hline & \multicolumn{2}{|c|}{ Congruent } & \multicolumn{2}{|c|}{ Incongruent } & & \\
\hline & RT & $S D$ & RT & $S D$ & & \\
\hline \multicolumn{7}{|l|}{ Across tasks (trial $n+1)$} \\
\hline Congruent & 638 & 13.6 & 720 & 14.0 & 82 & -7 \\
\hline Incongruent & 651 & 14.3 & 740 & 15.4 & 89 & \\
\hline \multicolumn{7}{|l|}{ Within task (trial $n+2)$} \\
\hline Congruent & 813 & 19.4 & 981 & 24.8 & 168 & $36^{*}$ \\
\hline Incongruent & 843 & 20.2 & 975 & 22.7 & 132 & \\
\hline \multicolumn{7}{|l|}{ Within task without stimulus priming } \\
\hline Congruent & 848 & 19.9 & 981 & 24.8 & 133 & -17 \\
\hline Incongruent & 843 & 20.2 & 993 & 23.8 & 150 & \\
\hline
\end{tabular}

Note. Values are reported in milliseconds. $N=42$. ${ }^{*} p<.05$.

\section{Discussion}

Experiment 1 yielded no evidence that dynamic changes in cognitive control could generalize across tasks: The presence of conflict in the Word task failed to modulate conflict resolution in the immediately following Number task. It is important that there was a small but statistically significant effect of incongruent words on overall RT: RTs to Number trials were $20 \mathrm{~ms}$ slower after an incongruent word than after a congruent word. Thus, there was statistical power to detect small cross-task modulation of congruency effect (i.e., $20 \mathrm{~ms}$ ), had such modulation been present.

Experiment 1 did reveal a dynamic trial $n$ to trial $n+2$ within-task modulation of conflict in the Word Stroop task, but this modulation was fully explained by repetition priming. This result replicates the findings of Mayr and collaborators (2003) in an alternating Flanker task. In that study, the stimuli alternated between vertical and horizontal displays, and participants had to alternate between up/down and right/left responses. As in our study, modulation was limited to trial $n+2$ and was fully explained by repetition priming (see also Verbruggen, Liefooghe, Notebaert, \& Vandierendonck, 2005). During repeated trials, the correct response is salient and there is little need for cognitive control. As a consequence, RTs are faster in trials with repeated stimuli. Thus, the enhanced conflict resolution that follows an incongruent trial appears to be caused not by "online" reactive adjustments of cognitive control but rather by a decrease in the cognitive demand; it seems that in these tasks, repeated stimuli bypass conflict altogether by increasing the salience of the correct response (Jacoby, Lindsay, \& Hessels, 2003; Mayr et al., 2003).

\section{Experiment $2 \mathrm{~A}$}

In Experiment 1, we explored dynamic trial-to-trial changes in cognitive control, whereas in Experiment 2A, we looked at more sustained modulations of cognitive control brought about by task demands. Trials alternated between the Word and the Number Stroop. The percentage of incongruent trials remained constant at $50 \%$ for the Number task, whereas the percentage of incongruent trials for the Word Stroop was either $25 \%$ or $75 \%$ in a block. We asked whether an increased percentage of incongruent trials in the Word task would reduce conflict in the Word task, the Number task, or both. In other words, we assessed within-task (word to

Table 2

Experiment 1 Percentages of Error Showing Cross- and Within-Task Trial-to-Trial Modulation Caused by the Word Stroop

\begin{tabular}{|c|c|c|c|c|}
\hline \multirow[b]{2}{*}{ Influence of Word Stroop (trial $n$ ) } & \multicolumn{2}{|c|}{ Probe trial } & \multirow{2}{*}{$\begin{array}{c}\text { Congruency } \\
\text { effect }\end{array}$} & \multirow[b]{2}{*}{ Modulation } \\
\hline & Congruent & Incongruent & & \\
\hline \multicolumn{5}{|l|}{ Across tasks (trial $n+1)$} \\
\hline Congruent & .5 & 5.4 & 4.9 & 0 \\
\hline Incongruent & .5 & 5.4 & 4.9 & \\
\hline \multicolumn{5}{|l|}{ Within task (Word: trial $n+2$ ) } \\
\hline Congruent & 2.2 & 6.2 & 4.0 & 1.5 \\
\hline Incongruent & 2.3 & 4.8 & 2.5 & \\
\hline \multicolumn{5}{|l|}{ Within task without stimulus priming } \\
\hline Congruent & 2.2 & 6.2 & 4.0 & 0.3 \\
\hline Incongruent & 2.3 & 6.0 & 3.7 & \\
\hline
\end{tabular}

Note. $\quad N=42$ 
word) and cross-task (word to number) sustained modulations of conflict resolution. We also assessed whether within-task sustained modulation could be fully explained by repetition priming. Finally, as in Experiment 1, we explored trial-to-trial dynamic fluctuations of the congruency effect and the role that repetition priming played in those fluctuations.

\section{Method}

Participants. Twenty students (12 women; mean age $=18.9$ years, $S D=1.0$, range $=18-21$ years) participated in the task. All but 2 participants were right-handed.

Stimulus and procedure. Stimuli, responses, and stimulusresponse assignments were the same as in Experiment 1 . There were two blocks of 288 trials. The percentage of incongruent Number trials was always $50 \%$, whereas the percentage of incongruent Word trials was $25 \%$ in one block and $75 \%$ in the other, counterbalanced across participants. At the beginning of each block, participants were informed about the ratio of congruent words and whether the block would be relatively easy (mostly congruent words) or relatively difficult (mostly incongruent words). We also told the participants that the ratio of congruent trials in the Number task would remain invariant. To control for the possible influence of instructions on participants' performance, we ran a second version of the experiment in which information about block difficulty and proportion of incongruent words was not provided. Data from that study yielded very similar results, including all the significant effects reported here (FernandezDuque \& Knight, 2006).

\section{Results}

Incorrect responses $(3.8 \%$ of Word trials and $3.1 \%$ of Number trials) and the two trials that immediately followed errors were excluded from the RT analyses. An additional $1.3 \%$ of trials were excluded due to no response, anticipatory responses $(\mathrm{RT}<200$ $\mathrm{ms}$ ), or unusually slow responses (RT $>2,500 \mathrm{~ms}$ for the Word task; RT $>1,500$ for the Number task). Error rates and mean RTs were calculated for task (Word, Number), trial type (congruent, incongruent), block type (75\% incongruent words, $25 \%$ incongruent words), and block order $(75 \% \rightarrow 25 \%, 25 \% \rightarrow 75 \%)$.

Absence of cross-task sustained modulation. We asked whether the proportion of incongruent trials in the Word Stroop modulated conflict resolution in the Number Stroop. As a means of testing this hypothesis, responses to the Number task were submitted to a mixed ANOVA with trial type (congruent, incongruent) and block type (75\% incongruent words, $25 \%$ incongruent words) as within-subject variables, and block order $(75 \% \rightarrow 25 \%, 25 \% \rightarrow 75 \%)$ as a betweensubjects variable. This analysis revealed that the congruency effect in the Number task was not modulated by the percentage of incongruent trials in the Word task (congruency effect in the block with mostly incongruent words: $78 \mathrm{~ms}$; congruency effect in the block with mostly congruent words: $77 \mathrm{~ms}$ ), $F(1,18)=0.6, M S E=164, n s$ (see Figure $3 \mathrm{~A})$. The same was true for the error data, $F(1,18)=0.03, M S E=$ 3.2, ns (see Tables 3 and 4).

Presence of within-task sustained modulation. According to the cognitive control literature, the congruency effect is reduced when incongruent trials occur very frequently (Gratton et al., 1992; Swick \& Jovanovic, 2002). To test this hypothesis, responses to

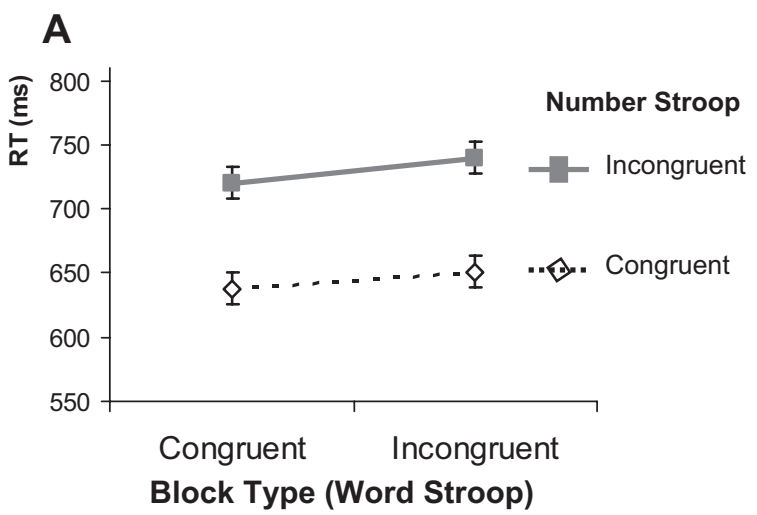

B
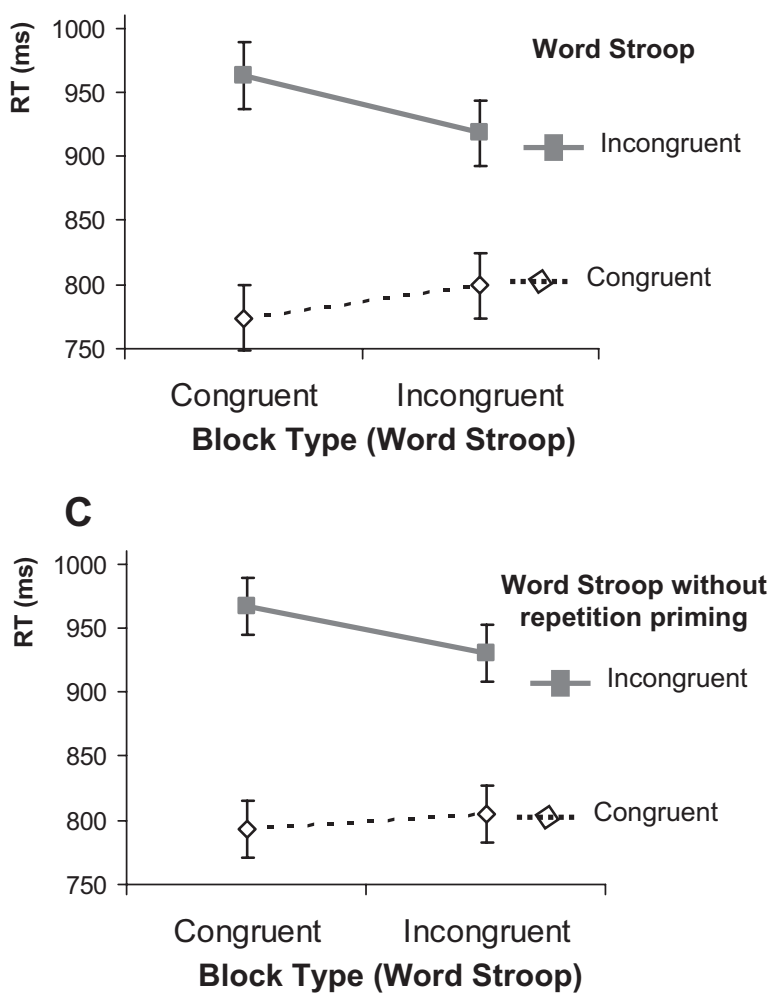

Figure 3. A-C: Sustained changes in congruency effect in Experiment $2 \mathrm{~A}$, as revealed by reaction times. Error bars show $95 \%$ confidence intervals based on the mean square error of the interaction, to partial out between-subjects variance (following Loftus \& Masson, 1994). A: Absence of cross-task modulation. The congruency effect in the Number Stroop was unaffected by block type. That is, whether the block had $75 \%$ incongruent words or $75 \%$ congruent words did not have an influence on the Number Stroop task. B: Presence of within-task modulation. The congruency effect in the Word Stroop was reduced in the block in which incongruent words occurred frequently, relative to the block in which most Word trials were congruent. C: Presence of within-task modulation in the absence of repetition priming. Filtering out trials in which the word-ink display was repeated did not eliminate the block type modulation of the Word Stroop congruency effect. 
Table 3

Experiment 2A Reaction Times (RT) Showing Cross- and Within-Task Block-Level Modulation Caused by the Word Stroop

\begin{tabular}{|c|c|c|c|c|c|c|}
\hline \multirow[b]{3}{*}{ Influence of Word Stroop } & \multicolumn{4}{|c|}{ Probe trial } & \multirow{3}{*}{$\begin{array}{c}\text { Congruency } \\
\text { effect }\end{array}$} & \multirow[b]{3}{*}{ Modulation } \\
\hline & \multicolumn{2}{|c|}{ Congruent } & \multicolumn{2}{|c|}{ Incongruent } & & \\
\hline & RT & $S D$ & RT & $S D$ & & \\
\hline \multicolumn{7}{|l|}{ Across tasks (Number) } \\
\hline Congruent $^{\mathrm{a}}$ & 599 & 17.0 & 677 & 18.8 & 78 & 1 \\
\hline Incongruent & 593 & 18.8 & 670 & 21.5 & 77 & \\
\hline \multicolumn{7}{|l|}{ Within task (Word) } \\
\hline Congruent & 774 & 29.0 & 963 & 32.1 & 189 & $70^{*}$ \\
\hline Incongruent & 799 & 36.5 & 918 & 35.3 & 119 & \\
\hline \multicolumn{7}{|c|}{ Within task without stimulus priming } \\
\hline Congruent & 793 & 30.2 & 967 & 32.4 & 174 & $49^{*}$ \\
\hline Incongruent & 805 & 37.4 & 930 & 35.3 & 125 & \\
\hline
\end{tabular}

Note. Values are reported in milliseconds. $N=20$.

a Denotes block type in which the Word Stroop was either mostly congruent or mostly incongruent.

${ }^{*} p<.05$

the Word task were submitted to a mixed ANOVA with trial type (congruent, incongruent) and block type (75\% incongruent words, $25 \%$ incongruent words) as within-subject variables, and block order $(75 \% \rightarrow 25 \%, 25 \% \rightarrow 75 \%)$ as between-subjects variable. Consistent with previous literature, congruency effect was smaller in the block with predominantly incongruent trials than in the block with predominantly congruent trials (189 ms vs. $119 \mathrm{~ms})$, $F(1,18)=7.9, M S E=3,088, p<.01$ (see Figure 3B). The error data showed a similar trend toward within-task modulation, though it failed to reach significance $(3.7 \%$ vs. $4.9 \%), F(1,18)=2.6$, $M S E=3.2, p<.12$.

Presence of within-task sustained modulation even in the absence of repetition priming. In Experiment 1, the task-specific improvement was largely due to stimulus repetition priming. To address whether repetition priming could also account for the modulation in Experiment 2A, we reanalyzed the data after filtering out trials with exact stimulus repetition. Even after filtering stimulus repetition trials, the congruency effect was reduced by the increased proportion of incongruent trials (125 ms vs. 174 $\mathrm{ms}), F(1,18)=5.3, M S E=2,239, p<.04$ (see Figure 3C). Next, we asked whether response priming could be the driving force behind the reduced congruency effect. To test this hypothesis, we filtered out trials in which the ink color (and thus the motor response) was repeated. Even after filtering out this type of priming, we found that the congruency effect was reduced by the increased proportion of incongruent trials (116 ms vs. $178 \mathrm{~ms}$ ), $F(1,18)=7.6, M S E=2,485, p<.01$. The error data failed to reveal a significant difference $(3.5 \%$ vs. $4.9 \%), F(1,18)=2.3$, $M S E=4.5, n s$.

Within-task trial-to-trial modulation and the role of repetition priming. Experiment 2A was designed to investigate block-level sustained adaptation in conflict resolution. Nonetheless, we also explored trial-to-trial adaptations, as we did in Experiment 1. Toward this goal, data from the Word Stroop task were submitted to a within-subject ANOVA with two variables: Word trial type (congruent, incongruent) and $n+2$ trial (congruent, incongruent). As in Experiment 1, conflict was reduced following an incongruent word (congruency effect following an incongruent word: $133 \mathrm{~ms}$;

Table 4

Experiment 2 A Percentages of Error Showing Cross- and Within-Task Block-Level Modulation Caused by the Word Stroop

\begin{tabular}{|c|c|c|c|c|}
\hline \multirow[b]{2}{*}{ Influence of Word Stroop } & \multicolumn{2}{|c|}{ Probe trial } & \multirow{2}{*}{$\begin{array}{l}\text { Congruency } \\
\text { effect }\end{array}$} & \multirow[b]{2}{*}{ Modulation } \\
\hline & Congruent & Incongruent & & \\
\hline \multicolumn{5}{|l|}{ Across task (Number) } \\
\hline Congruent $\mathrm{a}^{\mathrm{a}}$ & 0.8 & 5.1 & 4.3 & \multirow{2}{*}{-.3} \\
\hline Incongruent & 0.7 & 5.3 & 4.6 & \\
\hline \multicolumn{5}{|l|}{ Within task (Word) } \\
\hline Congruent & 1.7 & 5.6 & 4.9 & \multirow[t]{2}{*}{1.2} \\
\hline Incongruent & 1.7 & 5.4 & 3.7 & \\
\hline \multicolumn{5}{|c|}{ Within task without stimulus priming } \\
\hline Congruent & 0.6 & 5.7 & 5.1 & \multirow[t]{2}{*}{0.4} \\
\hline Incongruent & 1.9 & 6.7 & 4.8 & \\
\hline
\end{tabular}

Note. $\quad N=20$

${ }^{a}$ Denotes block type in which the Word Stroop was either mostly congruent or incongruent. 
congruency effect following a congruent word: $186 \mathrm{~ms}), F(1$, $19)=3.6, M S E=2,882, p<.07$. More important, this modulation disappeared after filtering out trials with repetition priming, $F(1,19)=0.2$, ns (congruency effect following an incongruent word: $161 \mathrm{~ms}$; congruency effect following a congruent word: 153 $\mathrm{ms}$ ). In other words, Experiment $2 \mathrm{~A}$ confirmed the causal role of repetition priming in trial-to-trial fluctuations of the congruency effect.

\section{Discussion}

Experiment $2 \mathrm{~A}$ revealed that conflict resolution could be modulated by the percentage of incongruent trials in a task. This sustained modulation was task specific and did not generalize to a different task despite the many similarities across the tasks, including the response overlap. This block-level modulation could not be explained as the aggregate effect of many trial-to-trial dynamic adaptations, nor could it be accounted for by repetition priming. Instead, it seems to reveal a genuine change in cognitive control brought about by task demands. The modulation of cognitive control at the block level contrasts with the lack of true modulation at the trial-to-trial level. Sustained adaptation in the absence of true trial-to-trial dynamic adaptation presents a challenge to theories posing the existence of a single mechanism for both effects, such as the conflict monitoring theory.

In Experiment 2A, the proportion of congruent or incongruent trials was confounded with increases or decreases in the frequency of the specific word-ink conjunctions. For example, a block with a high percentage of incongruent trials would also have a high percentage of "word green in blue ink" items, while those items would be relatively rare in a block with mostly congruent trials. This confound allows for an alternative explanation of the findings, based on an instance-based stimulus-response (S-R) learning model (Logan, 1988; Logan, Zbrodoff, \& Williamson, 1984). According to this view, each time a response is made to an item a trace is left in memory, and the accumulation of multiple traces of $\mathrm{S}-\mathrm{R}$ episodes facilitates responses to re-occurring items. Thus, RT to "word green in blue ink" is enhanced when displayed in a block with mostly incongruent trials not due to heightened cognitive control but rather due to past experience with the item and its response. A recent study investigating frequency effects in the Word Stroop supports this view (Jacoby, Lindsay, \& Hessels, 2003). In that study, half the colors were paired mostly with incongruent words, and the other half were paired mostly with congruent words. Thus, the overall proportion of congruent trials remained constant at $50 \%$. As predicted by associative learning, the congruency effect was smaller for the most frequent items. Just as that study manipulated item frequency while keeping constant the overall congruency ratio, it should be possible to vary the overall congruency ratio while keeping the frequency of certain items constant. We reasoned that this could be achieved by varying the ratio of congruent-to-incongruent trials for a subset of items, while keeping the ratio constant for another subset. We took this approach in Experiment 2B.

\section{Experiment 2B}

In Experiment 2B, we aimed to replicate Experiment 2A after eliminating the contribution of instance-based S-R learning. As in
Experiment 2A, the percentage of incongruent trials for the Word Stroop task was varied across blocks, but this time the congruent/ incongruent ratio of only two of the three colors changed across blocks. For the third color, the percentage of incongruent trials remained constant at 50\%. We tested whether this third color had a reduced congruency effect when occurring in the "mostly incongruent" block.

\section{Method}

Participants. Twenty-four students (17 women; mean age $=$ $18.8, S D=1.1$, range $=17-21)$ participated in the task. All but 1 participant were right-handed.

Stimulus and procedure. The paradigm was as in Experiment $2 \mathrm{~A}$, except for the following changes. The percentage of incongruent Word trials was $16.6 \%$ in one block and $83.3 \%$ in the other (in Experiment $2 \mathrm{~A}$ the percentages were $25 \%$ and $75 \%$ ). In the mostly incongruent block, two of the three colors were paired only with incongruent words (100\% incongruent). In the mostly congruent block, those two colors were paired only with congruent words (100\% congruent). The critical third color was paired with congruent words in $50 \%$ of the trials in either block. Which color served as the critical color was counterbalanced across participants.

\section{Results}

Incorrect responses (3.9\% of Word trials and 3.2\% of Number trials) and the two trials that immediately followed errors were excluded from the RT analyses. An additional 3.1\% of trials were excluded due to no response, anticipatory responses $(\mathrm{RT}<200$ $\mathrm{ms}$ ), or unusually slow responses (RT $>2,500 \mathrm{~ms}$ for the Word task; RT $>1,500$ for the Number task).

For two of the colors, only one type of trial was displayed in each block (either congruent or incongruent). Although those trials were very important in providing the context that presumably led to increases in cognitive control, performance differences in those trials were uninformative. Thus, these trials were not included in the analysis. ${ }^{2}$

Presence of within-task sustained modulation. To assess the modulation of the congruency effect, we looked at critical color trials. Those were trials displaying the color that had a constant ratio of congruency across blocks. To reduce practice and carryover effects, we filtered out the first 30 trials of each block. The averages for this dataset are displayed in Table 5 and Table 6 under the row heading "Within task (Word)." To prevent repetition priming from artificially reducing the congruency effect, we also filtered out trials with stimulus repetition. The averages for this data subset are in the rows labeled "Within task without stimulus priming" in Tables 5 and 6. Finally, to prevent the contribution of motor priming, we also filtered out trials in which the Word Stroop key press was the same as the key press for the preceding Number

\footnotetext{
${ }^{2}$ For these trials, mean RT was $740 \mathrm{~ms}(S D=131)$ for congruent trials and $916 \mathrm{~ms}(S D=132)$ for incongruent trials. Error rate was $1.9 \%(S D=$ 3.3 ) for congruent trials and $4.5 \%(S D=3.6)$ for incongruent trials.
} 
Table 5

Experiment 2B Reaction Times (RT) Showing Cross- and Within-Task Block-Level Modulation Caused by the Word Stroop

\begin{tabular}{|c|c|c|c|c|c|c|}
\hline \multirow[b]{3}{*}{ Influence of Word Stroop } & \multicolumn{4}{|c|}{ Probe trial } & \multirow{3}{*}{$\begin{array}{l}\text { Congruency } \\
\text { effect }\end{array}$} & \multirow[b]{3}{*}{ Modulation } \\
\hline & \multicolumn{2}{|c|}{ Congruent } & \multicolumn{2}{|c|}{ Incongruent } & & \\
\hline & $\mathrm{RT}$ & $S D$ & RT & $S D$ & & \\
\hline \multicolumn{7}{|l|}{ Across tasks (Number) } \\
\hline Congruent $^{\mathrm{a}}$ & 564 & 14 & 624 & 14 & 60 & -4 \\
\hline Incongruent & 577 & 12 & 641 & 13 & 64 & \\
\hline \multicolumn{7}{|l|}{ Within task (Word) } \\
\hline Congruent & 725 & 25 & 887 & 29 & 162 & 36 \\
\hline Incongruent & 812 & 27 & 938 & 29 & 126 & \\
\hline \multicolumn{7}{|c|}{ Within task without stimulus priming } \\
\hline Congruent & 735 & 26 & 900 & 30 & 165 & $49^{*}$ \\
\hline Incongruent & 831 & 29 & 947 & 29 & 116 & \\
\hline \multicolumn{7}{|c|}{$\begin{array}{l}\text { Within task with no stimulus priming } \\
\text { or motor priming }\end{array}$} \\
\hline Congruent & 715 & 26 & 907 & 31 & 192 & $80^{*}$ \\
\hline Incongruent & 822 & 32 & 934 & 30 & 112 & \\
\hline
\end{tabular}

Note. Values are reported in milliseconds. $N=24$.

a Denotes block type in which the Word Stroop was either mostly congruent or mostly incongruent.

${ }^{*} p<.05$.

Stroop. ${ }^{3}$ The averages for this final data subset are in rows labeled "Within task with no stimulus priming or motor priming." The decision to exclude all these trials was made a priori and was motivated by the goal to obtain a relatively pure measure of cognitive control. Thus, we describe here the statistical analyses performed on this data subset.

All participants had enough trials in each cell from which to compute reliable estimates of central tendency. The average number of trials per cell was 23.5, with a standard deviation of 3.5 and a range between 16 and 32 . For those trials, error rates and mean RTs were calculated for task (Word, Number), trial type (congruent, incongruent), block type ( $83 \%$ incongruent words, $17 \%$ incongruent words), and block order $(83 \% \rightarrow 17 \%, 17 \% \rightarrow 83 \%$; see Tables 5 and 6).

Responses to the Word task were submitted to a mixed ANOVA with trial type (congruent, incongruent) and block type (83\% incongruent words, $17 \%$ incongruent words) as within-subject variables and block order $(83 \% \rightarrow 17 \%, 17 \% \rightarrow 83 \%)$ as betweensubjects variable. The congruency effect was smaller in the block with predominantly incongruent trials than in the block with predominantly congruent trials (192 ms vs. $112 \mathrm{~ms}), F(1,22)=10.1$, $M S E=3,829, p<.001$. Overall RTs were slower in the block with predominantly incongruent trials than in the block with predominantly congruent trials, as revealed by a main effect of block type, $F(1,22)=7.8, M S E=1,373, p<.01$. This difference was modest ( $810 \mathrm{~ms}$ vs. $877 \mathrm{~ms}$ ), but it raised the possibility that differences in the congruency effect might be secondary to differences in overall speed of processing. To rule out this possibility, we computed proportional scores using congruent trials as the baseline. For each participant and each block, the congruency effect was divided by the mean RT for congruent trials. An analysis of the proportional scores confirmed that incongruent trials slowed down response to a larger extent when occurring among mostly congruent trials $(27.4 \%)$ than when occurring among mostly incongruent ones $(15.0 \%), F(1,22)=13.5, M S E=$ $138, p<.001$. Finally, the error data showed a similar trend toward within-task modulation, though it failed to reach significance $(3.0 \%$ vs. $6.3 \%), F(1,22)=2.5, M S E=3.2, p<.07$ (see Table 6).

Absence of cross-task sustained modulation. Although the main purpose of Experiment 2B was to explore sustained modulation within task, we also analyzed the data to assess the possibility of cross-task modulation. More specifically, we asked whether the proportion of incongruent trials in the Word Stroop modulated conflict resolution in the Number Stroop. As in the previous analysis, we excluded the first 30 trials of each block and trials with motor priming.

Responses to the Number task were submitted to a mixed ANOVA with trial type (congruent, incongruent) and block type (83\% incongruent words, $17 \%$ incongruent words) as withinsubject variables and block order $(83 \% \rightarrow 17 \%, 17 \% \rightarrow 83 \%)$ as between-subjects variable. As in Experiment 2A, this analysis revealed that the congruency effect in the Number task was not modulated by the percentage of incongruent trials in the Word task (congruency effect in the block with mostly incongruent words: 64 ms; congruency effect in the block with mostly congruent words: $60 \mathrm{~ms}), F(1,22)=0.5, M S E=227, n s$. The error data revealed a small but statistically significant difference, $F(1,22)=5.6$,

\footnotetext{
${ }^{3}$ There were not enough trials from which to compute reliable estimates of motor priming trials, and therefore we filtered these trials out instead of including motor priming as a variable in the ANOVA. Nonetheless, it is worth noting that motor priming trials revealed no congruency modulation. In the block with mostly congruent trials, mean RT for congruent trials was $764 \mathrm{~ms}$ and mean RT for incongruent trials was $889 \mathrm{~ms}$. In the block with mostly incongruent trials, mean RT for congruent trials was $851 \mathrm{~ms}$ and mean RT for incongruent trials was $973 \mathrm{~ms}$.
} 
Table 6

Experiment 2B Percentages of Error Showing Cross- and Within-Task Block-Level Modulation Caused by the Word Stroop

\begin{tabular}{|c|c|c|c|c|c|c|}
\hline \multirow[b]{3}{*}{ Influence of Word Stroop } & \multicolumn{4}{|c|}{ Probe trial } & \multirow{3}{*}{$\begin{array}{l}\text { Congruency } \\
\text { effect }\end{array}$} & \multirow[b]{3}{*}{ Modulation } \\
\hline & \multicolumn{2}{|c|}{ Congruent } & \multicolumn{2}{|c|}{ Incongruent } & & \\
\hline & $\%$ & $S D$ & $\%$ & $S D$ & & \\
\hline \multicolumn{7}{|l|}{ Across tasks (Number) } \\
\hline Congruent ${ }^{\mathrm{a}}$ & 1.0 & 0.3 & 7.0 & 1.1 & 6.0 & \multirow{2}{*}{$2.1^{*}$} \\
\hline Incongruent & 1.0 & 0.3 & 4.9 & 0.8 & 3.9 & \\
\hline \multicolumn{7}{|l|}{ Within task (Word) } \\
\hline Congruent & 2.2 & 1.0 & 7.7 & 1.3 & 5.5 & \multirow[t]{2}{*}{1.7} \\
\hline Incongruent & 2.8 & 0.8 & 6.6 & 1.3 & 3.8 & \\
\hline \multicolumn{7}{|c|}{ Within task without stimulus priming } \\
\hline Congruent & 2.4 & 1.0 & 8.2 & 1.4 & 5.8 & \multirow[t]{2}{*}{2.1} \\
\hline Incongruent & 2.7 & 0.8 & 6.4 & 1.3 & 3.7 & \\
\hline \multicolumn{7}{|c|}{$\begin{array}{l}\text { Within task with no stimulus priming } \\
\text { or motor priming }\end{array}$} \\
\hline Congruent & 2.4 & 1.1 & 8.7 & 1.6 & 6.3 & \multirow[t]{2}{*}{3.3} \\
\hline Incongruent & 2.9 & 0.9 & 5.9 & 1.2 & 3.0 & \\
\hline
\end{tabular}

${ }^{\text {a }}$ Denotes block type in which the Word Stroop was either mostly congruent or mostly incongruent.

${ }^{*} p<.05$.

$M S E=3.2, p<.05$. Incongruent trials in the Number task were more prone to errors in the block with mostly congruent words $(6.0 \%)$ than in the block with mostly incongruent words $(3.9 \%)$.

\section{Discussion}

Even when controlling for instance-based S-R learning, an increased ratio of incongruent trials led to a reduced congruency effect, consistent with a sustained increase in cognitive control. The absence of cross-task modulation revealed that setting separate levels of cognitive control for each task was possible. Given how similar the two tasks were in encoding, conflict resolution, motor preparation, and motor response, the lack of cross-task modulation may come as a surprise, particularly for theories that conceptualize conflict as an abstract entity (e.g., Botvinick et al., 2001). However, the result is consistent with theories stating that different task dimensions become activated or suppressed depending on their informational value (e.g., Egner \& Hirsch, 2005; Melara \& Algom, 2003). According to these latter theories, the frequent detection of conflict should increase the activation of the relevant dimension-color-as well as its goal representation ("attend to color").

Experiments 2A and 2B had long blocks of trials, and the trials alternated rapidly. Given these design features, it is unlikely that participants would consciously change the mindset for each trial based on previous trials or even based on the properties of the block. A more plausible scenario is that the modulation of cognitive control was brought about by task demands and without participants' overt intent. This raises the question of whether a paradigm that would encourage volitional changes in cognitive control would reveal a different pattern of results. We addressed this question in Experiments $3 \mathrm{~A}$ and $3 \mathrm{~B}$.

\section{Experiments $3 \mathrm{~A}$ and $3 \mathrm{~B}$}

In Experiment 3A, we assessed the voluntary modulation of cognitive control. Trials were displayed in sequences of three (word $\rightarrow$ number $\rightarrow$ word). At the beginning of each triplet sequence, a cue predicted with $100 \%$ accuracy the congruency of the Word Stroop trials, thus allowing participants to voluntarily establish the proper mindset. We assessed whether this voluntary modulation of cognitive control would generalize across tasks. In other words, we asked whether getting ready to process incongruent words would enhance conflict resolution in the Number Stroop task.

A finding of cross-task modulation with voluntary cueing in Experiment 3 would stand in contrast to the absence of cross-task modulation brought about by changes in task demands (Experiments 1 and 2). This contrast would be most striking if the cross-task modulation of voluntary cueing occurred in the absence of response overlap. Thus, two response modalities were used in Experiment 3: a verbal response to the Word task and a key press to the Number task. Also, to encourage participants to use a voluntary strategy, a long $(2,000 \mathrm{~ms})$ delay between the cue onset and the first word onset was used, and participants were allowed to start each triplet sequence whenever ready by pressing the space bar.

Experiment 3B was a control experiment to assess whether volitional cueing was truly necessary for cross-task modulation or whether the same results could be attributed to the conflict imposed by the incongruent word. If the cue was not necessary-if merely processing the incongruent word before the number task was sufficient for cross-task modulation - then the effect should be replicated without the cue information. To test this, we designed Experiment 3B to be identical to Experiment $3 \mathrm{~A}$ except that the cue was uninformative about the congruency of the upcoming trials.

\section{Method}

Participants. Twenty-five students participated in Experiment $3 \mathrm{~A}$ (12 women; mean age $=19.46, S D=1.05$, range $=18-22$ ). All but 2 participants were right-handed. Eighteen students (11 women; mean age $=19.4, S D=.94$, range $=19-22$ ) participated in Experiment 3B. All but 2 participants were right-handed. 
Apparatus and stimuli. For the Word Stroop, 16 stimuli were created by the combination of four words (red, blue, green, yellow) and font colors (red, blue, green, and yellow). For the Number Stroop, four digits $(1,2,3,4)$ were displayed in four different set sizes (1, 2, 3, and 4). Stimuli were displayed centrally in 18-point Courier New black font on a gray background. Thus, relative to the previous experiments, the size of the stimulus was reduced by half and the number of response choices was increased from three to four. These modifications were added to increase the task difficulty and thus foster the use of cognitive control. In Experiment 3A, the cues EASY and HARD were displayed for congruent and incongruent words, respectively. In Experiment 3B, the only cue was READY.

Procedure. Participants completed 80 sequences of three trials each (one Word trial, one Number trial, and one Word trial). After the participant started a sequence by pressing the space bar, a central cue (EASY, HARD) was displayed for 1,000 ms to warn the participant about the congruency of the following Word trials. The cue was $100 \%$ predictive for the two Word trials-which always had the same congruency-but was not predictive for the Number trial. After a 1,000-ms interval, the cue was followed by a Stroop word, and participants were instructed to name the color out loud. The Stroop word was always displayed for 1,000 ms. Five hundred ms later, the Stroop number was displayed and remained on the screen until the participant made a key response or for a maximum of 5,000 ms. Finally, after a 500-ms interval, another Stroop word appeared for 1,000 ms for participants to name out loud. At the end of the sequence, participants had up to $5 \mathrm{~s}$ to initiate the next sequence by pressing the space bar (see Figure 4).

Prior to beginning the experiment, all participants completed two practice sessions of 72 trials each, half of which were incongruent. The first practice session included only Number Stroop stimuli, and participants responded to the digit quantity by pressing $j, k, l$, and ; on a keyboard to indicate $1,2,3$, and 4, respectively. The second practice session included only Word Stroop stimuli, and participants responded to the font color of the word verbally into a microphone held by the researcher. Stimuli in both practice sessions remained on the screen for $5 \mathrm{~s}$ or until a response was given; the intertrial interval was $500 \mathrm{~ms}$.

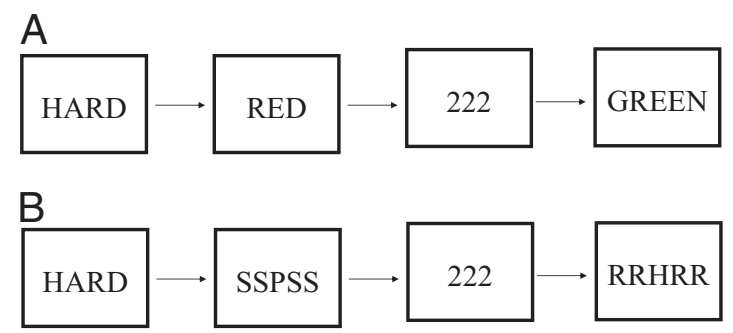

Figure 4. A: Design of Experiments 3A and 3B. The cue was $100 \%$ predictive for the Word Stroop: HARD was always followed by an incongruent word, whereas EASY was always followed by a congruent word (ink color is not illustrated by the figure but did follow the described rule in the actual experiment). In Experiment 3B, a noninformative cue was displayed (READY). B: Design of Experiment 4. Illustrated here is a case in which the cue predicted the occurrence of incongruent flanker. The cue was $100 \%$ predictive for the Flanker task: HARD was always followed by incongruent flankers, whereas EASY was always followed by congruent flankers.

\section{Results: Experiment $3 A$}

Incorrect responses (3.8\%) were excluded from the RT analyses. We also excluded trials with no response, anticipatory responses ( $\mathrm{RT}<200 \mathrm{~ms}$ ), or unusually slow responses $(\mathrm{RT}>1,500 \mathrm{~ms})$, as well as the first trial of each session (2\% of trials total). Error rates and mean RTs for the Number Stroop task were calculated based on trial type in the Number Stroop (congruent, incongruent) and cue type (easy, hard). Given that the cue was $100 \%$ predictive of the type of trial in the Word Stroop, the cue type was interchangeable with Word Stroop type.

As a means of assessing whether conflict resolution in the Number Stroop was enhanced by a mindset to process incongruent words, data from the Number Stroop task were submitted to a within-subject ANOVA with Number trial type (congruent, incongruent) and cue type (easy, hard) as variables. Unlike previous experiments in this article, the analysis revealed a significant cross-task modulation: When the Number Stroop trial was intermixed with incongruent words, the congruency effect of Number Stroop was significantly reduced (85 ms vs. $49 \mathrm{~ms}), F(1,24)=$ 7.0, $M S E=1,138, p<.01$ (see Figure 5A and Tables 7 and 8). There was no significant accuracy effect, $F(1,24)=0.6, M S E=$ $17, n s$. It is important that overall RTs for hard and easy cueing conditions were no different, $M_{\text {hard }}=634 \mathrm{~ms}, M_{\text {easy }}=644 \mathrm{~ms}$, $F(1,24)=2.5, M S E=1,008, n s$. This finding suggests that the reduction in congruency effect following the hard cue was not an artifact of overall response slowing.

\section{Results: Experiment $3 B$}

Incorrect responses (4\%) were excluded from the RT analyses. We also excluded trials with no response, anticipatory responses $(\mathrm{RT}<$ $200 \mathrm{~ms}$ ), or unusually slow responses (RT $>1,500 \mathrm{~ms}$ ), as well as the first trial of each session ( $0.5 \%$ of trials). Error rates and mean RTs for the Number Stroop task were calculated based on trial type in the Number Stroop (congruent, incongruent) and Word Stroop type (congruent, incongruent). Data were analyzed as in Experiment 3A.

Unlike Experiment $3 \mathrm{~A}$, in the absence of a cue to voluntarily engage in the task, there was no benefit of having an incongruent number surrounded by incongruent words (with congruent words: 54; with incongruent words: 61$), F(1,17)=0.2, M S E=1,023, n s$ (see Figure 5B). This result highlights the importance of the volitional component. There was no significant accuracy effect, $F(1,17)=0.3, M S E=14, n s$

\section{Discussion}

In Experiment 3A we found for the first time in our study that enhanced cognitive control generalized across tasks. This finding was all the more striking in that the two tasks had different modalities of response (verbal, motor). The difference in response modality argues against interference at the effector level and favors a common central processor shared by the two tasks. This is in marked contrast with the findings of Experiment 2, which revealed separate control settings for each task when changes were triggered by task demands.

Experiment 3B pointed out the critical role that voluntarily attending to predictive cues plays in such cross-task modulation. When the cue was uninformative, the cross-task modulation disappeared. Thus, engaging in conflict resolution in itself was not 

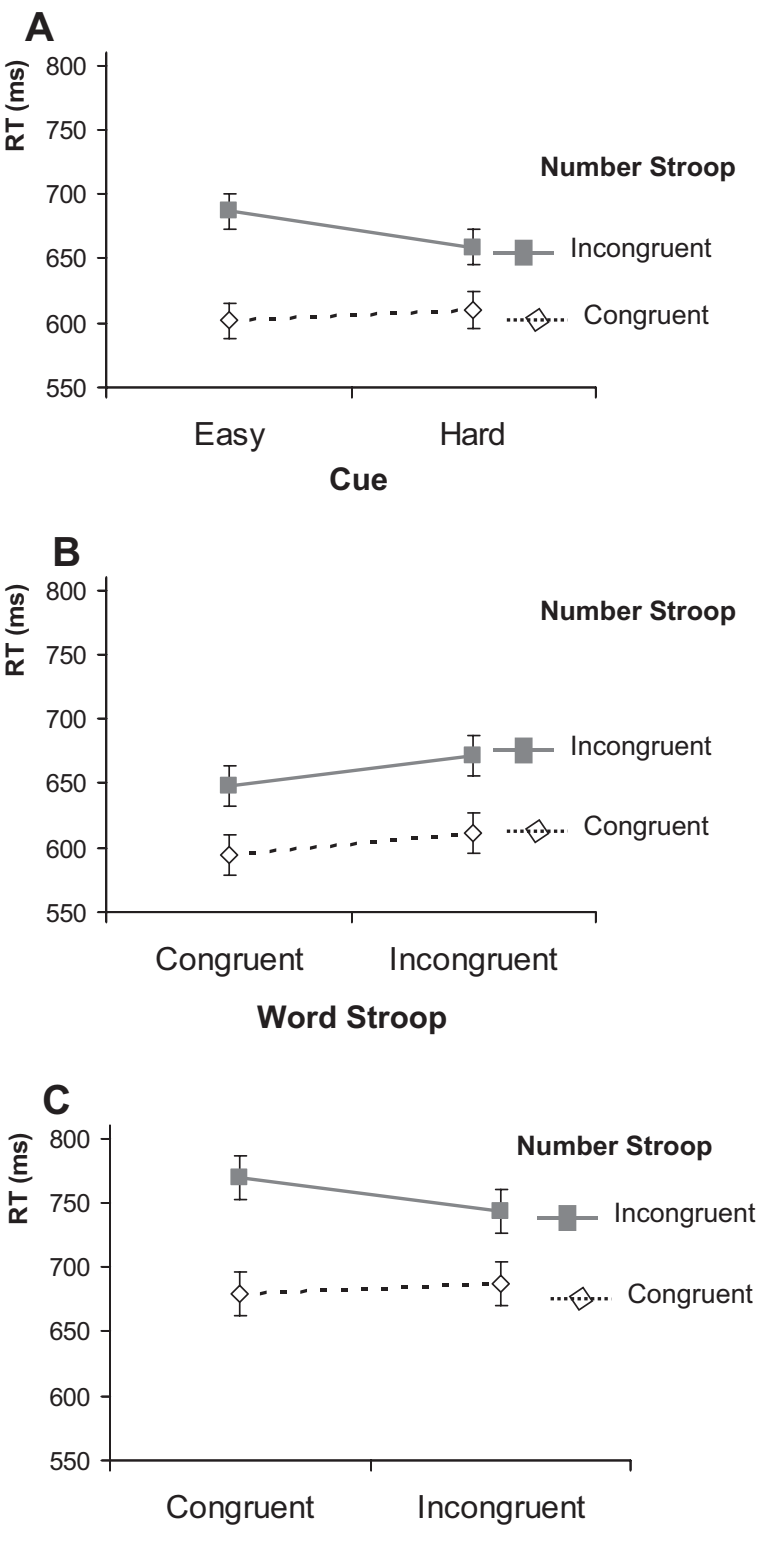

Flanker task

Figure 5. Voluntary changes in congruency effect as revealed by reaction times (RT) in Experiments 3A, 3B, and 4. Error bars show 95\% confidence interval based on the mean square error of the interaction, to partial out between-subjects variance (following Loftus \& Masson, 1994). A: Presence of cross-task modulation following a predictive cue. In Experiment $3 \mathrm{~A}$, the cue EASY was always followed by a congruent word. The congruency effect in the Number Stroop was reduced following a cue (HARD) that predicted the incongruency of the Word Stroop trials. B: Absence of cross-task modulation following a nonpredictive cue. The congruency effect in the Number Stroop was not modified following a nonpredictive cue (READY) in Experiment 3B. C: Presence of cross-tasks modulation following a predictive cue for a Flanker task. In Experiment 4, the cue EASY was always followed by congruent flankers. The congruency effect in the Number Stroop was reduced following a cue (HARD) that predicted the incongruency of the Flanker trials. sufficient for cross-task modulation, a result that is consistent with Experiments 1 and 2.

In Experiment 3A, getting ready to process incongruent words reduced the congruency effect in the Number Stroop task. We interpreted this result to suggest that increased cognitive control for the Word task was carried over to the Number task. However, there is an alternative interpretation that requires no modulation of cognitive control and thus would challenge our main conclusion: The EASY cue may have biased participants to read the word rather than name its color. After reading the word, participants would be biased to continue reading (i.e., reporting the identity of the digit), thus increasing the cost when the number identity conflicted with the quantity. Also, the cue warning about the occurrence of an incongruent word might have biased participants to look at the stimulus parafoveally or to defocus their eyes. Any of these alternatives could account for the findings of Experiment 3A. In Experiment 4, we designed a paradigm that was immune to these alternative accounts.

\section{Experiment 4: Flanker Task}

In Experiment 4, we aimed to replicate Experiment 3 using a Flanker task instead of a Word Stroop task. In the Flanker task, participants responded to the central item while ignoring the peripheral distractors. The distractors could be congruent or incongruent with the central target. As in Experiment 3A, trials were displayed in sequences of three (flanker $\rightarrow$ number $\rightarrow$ flanker). At the beginning of each triplet sequence, a cue predicted with $100 \%$ accuracy the congruency of the Flanker trials, thus allowing participants to voluntarily establish the proper mindset.

We reasoned that, unlike in the Word Stroop task of Experiment 3 , the "reading strategy" would not be disproportionately applied to congruent trials in the Flanker task. We also reasoned that unlike incongruent word trials, incongruent Flanker trials would bias participants to focus their attention at the center of the screen. Thus, if a cue warning about an incongruent flanker enhanced conflict resolution in the Number Stroop, we could rest assured that the effect was not caused by a peripheral strategy such as parafoveal looking or eye defocusing.

\section{Method}

Participants. Data from 25 students were collected for Experiment 4. Two other participants were excluded because their performance was close to chance levels, suggesting that they had not followed the instructions.

Stimuli and procedure. The same stimuli as in Experiment 3A were used for the Number Stroop task in Experiment 4. For the Flanker task, each of four letters (H, R, S, P) was combined with itself to create congruent trials (HHHHH) and with one another to create incongruent trials (RRHRR, SSHSS, PPHPP). The same four keys $(j, k, l, ;)$ were assigned to the Number task and the Flanker task, thus creating response overlap across tasks. In either task, the target stimulus remained until response or for a maximum of 5,000 ms, with a response-stimulus interval of $1,500 \mathrm{~ms}$. Participants completed 96 sequences of three trials each (one Flanker trial, one Number trial, and one Flanker trial). There were 24 trials per each condition of interest. All other aspects of the design were identical to those in Experiment 3A. 
Table 7

Experiments $3 A$ and $3 B$ Reaction Times (RT) Showing the Effect of Cued Word Stroop on the Number Stroop

\begin{tabular}{|c|c|c|c|c|c|c|}
\hline \multirow[b]{3}{*}{ Cue: Word Stroop type } & \multicolumn{4}{|c|}{ Number Stroop } & \multirow{3}{*}{$\begin{array}{c}\text { Congruency } \\
\text { effect }\end{array}$} & \multirow[b]{3}{*}{ Modulation } \\
\hline & \multicolumn{2}{|c|}{ Congruent } & \multicolumn{2}{|c|}{ Incongruent } & & \\
\hline & RT & $S D$ & RT & $S D$ & & \\
\hline \multicolumn{7}{|l|}{ Experiment $3 \mathrm{~A}$} \\
\hline EASY congruent & 602 & 21.4 & 687 & 24.2 & 85 & $36^{*}$ \\
\hline HARD incongruent & 610 & 22.9 & 659 & 20.9 & 49 & \\
\hline \multicolumn{7}{|l|}{ Experiment $3 \mathrm{~B}$} \\
\hline READY congruent & 594 & 22.0 & 648 & 18.9 & 54 & -7 \\
\hline READY incongruent & 611 & 26.0 & 672 & 26.2 & 61 & \\
\hline
\end{tabular}

Note. Values are reported in milliseconds. Experiment $3 \mathrm{~A}, N=25$. Experiment $3 \mathrm{~B}, N=18$.

${ }^{*} p<.05$

\section{Results}

Incorrect responses (2.3\%) were excluded from the analysis. We also excluded the first trial of each block; error trials to the first word of the sequence; and trials with no response, anticipatory responses $(\mathrm{RT}<200)$, and unusually slow responses $(\mathrm{RT}>$ 1,500). A total of $6.3 \%$ of trials were excluded for these reasons. Finally, we excluded trials with motor priming (i.e., trials in which the motor response to the Flanker task was the same as for the Number Stroop). ${ }^{4}$ Data were analyzed in the same way as in Experiment $3 \mathrm{~A}$ and yielded same pattern of results.

Error rates and mean RTs for the Number Stroop task were calculated based on trial type in the Number Stroop (congruent, incongruent) and cue type (easy, hard). Given that the cue was $100 \%$ predictive of the type of trial in the Flanker task, cue type was interchangeable with Flanker type. To assess whether conflict resolution in the Number Stroop was enhanced by a mindset to process incongruent flankers, data from the Number Stroop task were submitted to a within-subject ANOVA with Number trial type (congruent, incongruent) and cue type (easy, hard) as variables. As in Experiment $3 \mathrm{~A}$, the analysis revealed significant cross-task modulation: When the Number Stroop trial was intermixed with incongruent flankers, the congruency effect of Number Stroop was significantly reduced (91 ms vs. $57 \mathrm{~ms}), F(1,24)=$ 4.2, $M S E=1,727, p<.05$ (see Figure 5c and Table 9). Overall RTs for hard and easy cueing conditions were no different $\left(M_{\text {hard }}=716 \mathrm{~ms}, M_{\text {easy }}=724 \mathrm{~ms}\right), F(1,24)=0.9, M S E=2,008$, $n s$. This finding suggests that the reduction in congruency effect following the HARD cue was not an artifact of overall response slowing. The error data revealed a main congruency effect, $F(1$, $24)=10.7, M S E=23, p<.003$, but no significant interaction, $F(1,24)=.9, M S E=9, n s$.

Finally, we explored the Flanker task data. In a within-subject ANOVA we entered as variables cue type (easy, hard), trial position in the sequence (first, last), and Number trial type (congruent, incongruent). Cue type was $100 \%$ predictive of conflict. The presence of conflict in the Flanker task (i.e., hard cue trials) slowed down response by $81 \mathrm{~ms}$ (934 ms vs. $1,015 \mathrm{~ms}), F(1,23)=27.0, M S E=10,353, p<$ $.001 .^{5}$ The effect was larger for the first trial in the sequence than for the last one, as revealed by the Cue Type $\times$ Trial Position interaction, $F(1,23)=4.6, M S E=3,813, p<.05$. More important for the purpose of this study, whether the Number trial was congruent or incongruent had no effect on the Flanker task $(p>.20)$.

\section{Discussion}

The main finding of Experiment 3A was replicated in Experiment 4: A cue warning participants to process conflict in one task (i.e., the Flanker task) led to reduced conflict in the other (i.e., the Number Stroop). The finding could not be explained by a "reading" strategy, as reading was necessary both for congruent and incongruent trials of the Flanker task. In this regard, the Flanker task differed from the Word Stroop task of Experiment 3A, in which reading was useful only for congruent trials. Neither could the finding be explained by the use of a "peripheral strategy." Unlike the Word Stroop task, incongruent Flanker trials should bias participants to focus their attention at the center of the screen. Thus, the replication of Experiment 3A using a Flanker task ruled out those alternative interpretations. The generalization to a task structurally similar to the Number Stroop should allow future studies to systematically manipulate different properties of the Flanker task (e.g., response vs. stimulus conflict) to uncover the mechanisms underlying cross-task modulation of cognitive control. The results from this experiment suggest that the effect may occur at later stages of information processing than previously speculated (Botvinick et al., 2001; Gratton et al., 1992).

\footnotetext{
${ }^{4}$ In approximately $25 \%$ of trials, the motor response to the number task was the same as to the preceding Flanker task (i.e., motor priming). We filtered these trials out rather than including motor priming as a variable in the analysis because there were not enough trials from which to compute reliable estimates, with some participants having as few as three trials. However, it is worth noting that in trials with motor priming we did not find cue modulation. Following the EASY cue, mean RT for congruent trials was $704 \mathrm{~ms}(S D=120)$, and mean RT for incongruent trials was 752 $\mathrm{ms}(S D=133)$. Following the HARD cue, mean RT for congruent trials was $649(S D=107)$, and mean RT for incongruent trials $734 \mathrm{~ms}(S D=$ 129)
}

${ }^{5} \mathrm{RT}$ data were not collected for 1 participant due to technical error. 
Table 8

Experiments $3 A$ and $3 B$ Percentages of Error Showing the Effect of Cued Word Stroop on the Number Stroop

\begin{tabular}{lccccc}
\hline & \multicolumn{2}{c}{ Number Stroop } & & \\
\cline { 2 - 3 } Cue: Word Stroop type & Congruent & Incongruent & & $\begin{array}{c}\text { Congruency } \\
\text { effect }\end{array}$ & Modulation \\
\hline Experiment 3A & & & & \\
$\quad$ EASY congruent & 1.2 & 7.1 & 5.9 & 1.4 \\
$\quad$ HARD incongruent & 1.4 & 5.9 & & \\
Experiment 3B & & & & 0.3 \\
$\quad$ READY congruent & 0.6 & 5.7 & 5.1 & \\
READY incongruent & 1.9 & 6.7 & 4.8 & \\
\hline
\end{tabular}

Note. Experiment 3A, $N=25$. Experiment 3B, $N=18$.

\section{General Discussion}

In four experiments, we asked whether fluctuations in cognitive control were the property of a single supervisory system. We hypothesized that the answer to this question would vary depending on whether cognitive control was modulated by changes in task demand or by participants' voluntary use of informative cues. At a more basic level, we asked whether reductions in congruency effect were a true reflection of increased cognitive control or merely an artifact of repetition priming. We first address this basic question of repetition priming.

Experiment 1 revealed dynamic changes in congruency effect that were task specific and fully explained by repetition priming. This finding serves as a cautionary note that priming effects should be tested before reactive changes in cognitive control can be concluded (Mayr et al., 2003). This being said, other studies have shown online modulations of cognitive control even after controlling for repeated trials. What may lie at the core of this discrepancy? One factor that may contribute to differences across studies is the inclusion of partial repetition trials. These are trials in which one feature is repeated and the other feature is changed (e.g., the same word presented in a different color than in the preceding trial). Partial repetitions usually lead to slower RTs than either complete repetitions or complete alternations, presumably because stimulus and response features are temporarily associated in each trial, and partial repetition trials violate that association (Hommel,
1998; Hommel, Proctor, \& Vu, 2004; Notebaert \& Soetens, 2003; Wendt, Kluwe, \& Peters, 2006). However, partial repetitions cannot fully explain the disagreement, because some studies have reported the presence of sequential effects even when controlling for partial repetitions (Kerns et al., 2004; Kunde \& Wühr, 2006; Notebaert et al., 2006).

Another factor that may account for different findings across studies is the pace at which incongruent stimuli are displayed. In Eriksen's Flanker task, in which participants have to respond to the central item while ignoring the distracting flankers, modulation of cognitive control has been found when using an intertrial interval of $10 \mathrm{~s}$ (Botvinick et al., 1999), 5-6 s (Ullsperger et al., 2005), or 3.5-5.5 s (Gratton et al., 1992) but not when using a responsestimulus interval of $1 \mathrm{~s}$ (Mayr et al., 2003). In the Word Stroop task, reactive modulation of cognitive control has been observed when using a stimulus-stimulus interval of $3 \mathrm{~s}$ (Kerns et al., 2004) but not when using a response-stimulus interval of $500 \mathrm{~ms}$ (Experiment 1 of the current study). A slow pace of stimulus presentation may encourage participants to use a voluntary modulation of cognitive control on trial-to-trial bases, whereas fast-paced studies are likely to discourage such an effortful strategy. A slow pace may also reduce the amount of priming, thus revealing true effect on cognitive modulation. The distinction between slow and fast pace is particularly important for the interpretation of neuroimaging studies, which in general have used long intertrial intervals, as required by

Table 9

Experiment 4 Mean Reaction Times (RT) and Percentages of Error Showing the Effect of Cued Flanker Task on the Number Stroop

\begin{tabular}{|c|c|c|c|c|c|c|}
\hline \multirow[b]{3}{*}{ Cue: Flanker type } & \multicolumn{4}{|c|}{ Number Stroop } & \multirow{3}{*}{$\begin{array}{l}\text { Congruency } \\
\text { effect }\end{array}$} & \multirow[b]{3}{*}{ Modulation } \\
\hline & \multicolumn{2}{|c|}{ Congruent } & \multicolumn{2}{|c|}{ Incongruent } & & \\
\hline & $\mathrm{RT}$ & $S D$ & RT & $S D$ & & \\
\hline \multicolumn{7}{|l|}{ Mean RT } \\
\hline EASY congruent & 679.0 & 22.0 & 770.0 & 20.2 & 91.0 & $34.0^{*}$ \\
\hline HARD incongruent & 687.0 & 23.1 & 744.0 & 23.7 & 57.0 & \\
\hline \multicolumn{7}{|l|}{ Error rate } \\
\hline READY congruent & 0.8 & 0.4 & 3.4 & 1.1 & 2.8 & -0.9 \\
\hline READY incongruent & 0.6 & 0.4 & 4.3 & 1.2 & 3.7 & \\
\hline
\end{tabular}

Note. Reaction time values are reported in milliseconds. Experiment $4, N=25$.

${ }^{*} p<.05$. 
technical constraints in extracting the hemodynamic signal. However, differences in intertrial interval cannot provide a full explanation, as some studies have found a trial-to-trial modulation in the absence of repetition priming, even at short intervals (Notebaert et al., 2006).

Although dynamic cross-task trial-to-trial modulation of cognitive control was absent in Experiment 1, a sustained increase in top-down regulation was observed in Experiment 2; congruency effect was reduced in blocks with a high ratio of incongruent-to-congruent trials. More important, this modulation survived the filtering of repetition trials. To our knowledge, ours is the first experiment to control for repetition priming in paradigms that use congruency ratio to modulate congruency effect. Given the wide use of such a paradigm in studies of top-down control, it was welcome news that the effect is genuine (Gratton et al., 1992; Naccache et al., 2005; Swick \& Jovanovic, 2002). On the other hand, although the effect was not fully explained by priming, priming did partly contribute to the reduction in congruency effect. Thus, future studies should continue filtering repetition priming to generate an accurate estimate of the true cognitive modulation. Furthermore, researchers should be mindful of other stimulus and response properties that may contribute to fluctuations in the congruency effect, even in the absence of top-down modulation. One such factor is the frequency with which specific items are displayed. As shown in Experiment 2B, cognitive control can be modulated by task demand even under conditions in which instance-based learning is not a factor. However, this requires experimental designs that pay special attention to variations in item frequency. Partial alternations and repetitions of higher order features are two other factors that may contribute to bottom-up fluctuations in congruency effect (Hommel et al., 2004; Mayr et al., 2003).

Experiment 2 also shed light on the question of task specificity in cognitive control modulation. We asked whether it was possible to set separate levels of cognitive control for rapidly alternating tasks. As a strong test of this hypothesis, we chose two tasks similar in their encoding, locus of conflict resolution, and response output. Despite these similarities, increases in task demand triggered a top-down modulation only in the specific task. Some recent studies relate to these present findings. In one study, Eriksen's Flanker task was used to explore the existence of separate cognitive control systems in each hemisphere (Corballis \& Gratton, 2003). The proportion of incongruent trials was manipulated across visual fields. A large probability of incongruent trials in one visual field reduced the congruency effect, but this benefit did not generalize to the visual field in which incongruent trials were rare, a result consistent with the existence of two parallel control systems (see also Awh, Matsukura, \& Serences, 2003). The use of spatial context for the modulation of cognitive control has also been observed in the Stroop task (Crump, Gong, \& Milliken, 2006). In a modified version of the classic paradigm, a prime word was displayed at fixation followed by a colored rectangle above or below fixation. The task was to report the color of the rectangle, and congruency between prime and rectangle was manipulated by location. Conflict was reduced at the location in which incongruent trials were frequent. These studies show it is possible to establish separate levels of cognitive control based on spatial information. Our Experiment 2 revealed that separate levels of cognitive control can be established based on temporal and task information. Together, the studies argue for a fair degree of specificity at the level of cognitive control. Thus, while the conflict monitoring system may act at a high level of abstraction, accepting varied types of inputs and providing a single common output (perceived difficulty), regulation by the cognitive control system appears to be task specific when triggered by task demands. The presence of task-specific modulation is consistent with theories stating that different task dimensions become activated or suppressed depending on their informational value (e.g., Melara \& Algom, 2003). According to these theories, conflict detection leads to an increase in working memory of task-specific goal representations. Recent neuroimaging data provide support for this view (Egner \& Hirsch, 2005). An important question for future research would be to specify the mechanism by which a nonspecific output of perceived conflict gets channeled to a specific control system.

The pattern of results when cognitive control was endogenously generated was quite different from the pattern when cognitive control was enhanced by task demands: Getting ready to process conflict in one task did reduce conflict in another task (Experiments 3 and 4). Merely engaging in conflict resolution was not sufficient (Experiment 3B): A combination of a prepared mind and active engagement was required (Experiments $3 \mathrm{~A}$ and 4 ). The modulation occurred across tasks that had different input (words, numbers) and different output (verbal, motor), providing strong evidence against a repetition priming account. These results highlight the distinction between volitional and task-dependent modulations of cognitive control, a distinction that has not been prominently featured in previous computational models. An important goal for future research would be to uncover the mechanisms underlying those differences. One possibility is that a central supervisory system may be recruited for establishing the top-down influence (Experiments $3 \mathrm{~A}$ and 4), but not for maintaining that influence once it has been established (Experiment 2). This interpretation would be consistent with visual search studies that show dual-task interference across domains (visual, auditory) in establishing a mental set of "to-be-ignored" items, but only domainspecific interference in maintaining the set (Humphreys, Watson, \& Jolicoeur, 2002). Alternatively, cross-task modulation may depend on the stage of information processing at which conflict is resolved. In this regard, the paradigm introduced in Experiment 4 may be particularly useful. The structural similarity between the Flanker task and the Number Stroop should allow the systematic manipulation of cognitive control in one task and its assessment in the other. By varying the stage at which conflict occurs (i.e., response vs. stimulus), it should be possible to explore volitional cross-task modulation of conflict at perceptual and motoric levels. Doing so would further our understanding of the commonalities and specificities of cognitive control.

\section{References}

Awh, E., Matsukura, M., \& Serences, J. T. (2003). Top-down control over biased competition during covert spatial orienting. Journal of Experimental Psychology: Human Perception and Performance, 29(1), 52-63.

Botvinick, M. M., Braver, T. S., Barch, D. M., Carter, C. S., \& Cohen, J. D. (2001). Conflict monitoring and cognitive control. Psychological Review, 108, 624-652.

Botvinick, M., Nystrom, L., Fissell, K., Carter, C. S., \& Cohen, J. D. (1999). Conflict monitoring versus selection-for-action in anterior cingulated cortex. Nature, 402, 179-181.

Bush, G., Luu, P., \& Posner, M. I. (2000). Cognitive and emotional influences in anterior cingulate cortex. Trends in Cognitive Sciences, 4(6), 215-222. 
Bush, G., Whalen, P. J., Rosen, B. R., Jenike, M. S., McInerney, S. C., \& Rauch, S. L. (1998). The counting Stroop: An interference task specialized for function neuroimaging: Validation study with function MRI. Human Brain Mapping, 6, 270-282.

Corballis, P. M., \& Gratton, G. (2003). Independent control of processing strategies for different locations in the visual field. Biological Psychology, 64, 191-209.

Crump, M. J. C., Gong, Z., \& Milliken, B. (2006). The context-specific proportion of congruent Stroop effect: Location as a contextual cue. Psychonomic Bulletin \& Review, 13(2), 316-321.

Durgin, F. H. (2000). The reverse Stroop effect. Psychonomic Bulletin \& Review, 7, 121-125.

Egner, T., \& Hirsch, J. (2005). Cognitive control mechanisms resolve conflict through cortical amplification of task-relevant information. $\mathrm{Na}$ ture Neuroscience, 8, 1784-1790.

Fernandez-Duque, D., \& Knight, M. (2006). [Sustained modulation of cognitive control]. Unpublished raw data.

Gratton, G., Coles, M. G. H., \& Donchin, E. (1992). Optimizing the use of information: Strategic control of activation and responses. Journal of Experimental Psychology: General, 121, 480-506.

Hommel, B. (1998). Event files: Evidence for automatic integration of stimulus-response episodes. Visual Cognition, 5, 183-216.

Hommel, B., Proctor, R. W., \& Vu, K-P. L. (2004). A feature-integration account of sequential effects in the Simon task. Psychological Research, $68,1-17$.

Humphreys, G. W., Watson, D. G., \& Jolicoeur, P. (2002). Fractionating the preview benefit: Dual-task decomposition of visual marking by timing and modality. Journal of Experimental Psychology: Human Perception and Performance, 28, 2-42.

Jacoby, L. L., Lindsay, D. S., \& Hessels, S. (2003). Item-specific control of automatic processes: Stroop process dissociations. Psychonomic Bulletin \& Review, 10, 638-644.

Kerns, J., Cho, R., Stenger, V., Cohen, J., MacDonald, A., \& Carter, C. (2004). Anterior cingulate monitoring and adjustments in control. Science, 303, 1023-1026.

Kunde, W., \& Wühr, P. (2006). Sequential modulations of correspondence effects across spatial dimensions and tasks. Memory \& Cognition, 34(2), 356-367.

Loftus, G. R., \& Masson, M. E. (1994). Using confidence intervals in within-subject designs. Psychonomic Bulletin \& Review, 1, 476-490.

Logan, G. D. (1985). Executive control of thought and action. Acta Psychologica, 60, 193-210.

Logan, G. D. (1988). Toward an instance theory of automization. Psychological Review, 95, 492-527.

Logan, G. D., \& Bundesen, C. (2003). Clever Homunculus: Is there an endogenous act of control in the explicit task-cuing procedure? Journal of Experimental Psychology: Human Perception and Performance, 29(3), 575-599.

Logan, G. D., \& Zbrodoff, N. J. (1979). When it helps to be misled: Facilitation effects of increasing the frequency of conflicting stimuli in a Stroop-like task. Memory and Cognition, 7, 166-174.

Logan, G. D., Zbrodoff, N. J., \& Williamson, J. (1984). Strategies in the color-word Stroop task. Bulletin of the Psychonomic Society, 22, 135-138.

MacDonald, A. W., Cohen, J. D., Stenger, V. A., \& Carter, C. S. (2000). Dissociating the role of the dorsolateral prefrontal cortex and anterior cingulate cortex in cognitive control. Science, 288, 1835-1838.

MacLeod, C. M. (1991). Half a century of research on the Stroop effect: An integrative review. Psychological Bulletin, 109(2), 163-203.

Mayr, U., Awh, E., \& Laurey, P. (2003). Conflict adaptation effects in the absence of executive control. Nature Neuroscience, 6, 450-452.

Melara, R. D., \& Algom, D. (2003). Driven by information: A tectonic theory of Stroop effects. Psychological Review, 110, 422-471.

Miyake, A., Friedman, N. P., Emerson, M. J., Witzki, A. H., \& Howerter,
A. (2000). The unity and diversity of executive functions and their contribution to complex "frontal lobe" tasks: A latent variable analysis. Cognitive Psychology, 41, 49-100.

Naccache, L., Dehaene, S., Cohen, L., Habert, M-O., Guichart-Gomez, E., Galanaud, D., \& Willer, J.-C. (2005). Effortless control: Executive attention and conscious feeling of mental effort are dissociable. Neuropsychologia, 43, 1318-1328.

Notebaert, W., Gevers, W., Verbruggen, F., \& Liefooghe, B. (2006). Top-down and bottom-up sequential modulations of congruency effects. Psychonomic Bulletin \& Review, 13(1), 112-117.

Notebaert, W., \& Soetens, E. (2003). The influence of irrelevant stimulus changes on stimulus and response repetition effects. Acta Psychologica, $112(2), 143-156$

Notebaert, W., Verbruggen, F., \& Soetens, E. (2005). Sequential analysis of relevant and irrelevant stimulus information in the Stroop task. European Journal of Cognitive Psychology, 17, 642-658.

Posner, M. I., \& DiGirolamo, G. J. (1998). Executive attention: Conflict, target detection, and cognitive control. In R. Parasuraman (Ed.), The attentive brain (pp. 401-423). Cambridge, MA: MIT Press.

Posner, M. I., \& Fernandez-Duque, D. (1999). Attention in the human brain. In R. A. Wilson \& F. C. Keil (Eds.), The MIT encyclopedia of the cognitive sciences (pp. 43-46). Cambridge, MA: MIT Press.

Raz, A., Shapiro, T., Fan, J., \& Posner, M. I. (2002). Hypnotic suggestion and the modulation of Stroop interference. Archives of General Psychiatry, 59, 1155-1161.

Reisberg, D., Baron, J., \& Kemler, D. G. (1980). Overcoming Stroop interference: The effects of practice on distractor potency. Journal of Experimental Psychology: Human Perception and Performance, 6(1), $140-150$.

Ridderinkhof, K. R., Ullsperger, M., Crone, E. A., \& Nieuwenhuis, S. (2004). The role of medial frontal cortex in cognitive control. Science, 306, 443-447.

Rueda, M. R., Fan, J., McCandliss, B. D., Halparin, J. D., Gruber, D. B., Lercari, L. P., \& Posner, M. I. (2004). Development of attentional networks in childhood. Neuropsychologia, 42(8), 1029-1040.

Stroop, J. R. (1935). Studies of interference in serial verbal reactions. Journal of Experimental Psychology: General, 18, 643-662. (Reprinted 1992, Journal of Experimental Psychology: General, 122, 15-23).

Stürmer, B., Leuthold, H., Soetens, E., Schröter, H., \& Sommer, W. (2002). Control over location-based response activation in the Simon task: Behavioral and electrophysiological evidence. Journal of Experimental Psychology: Human Perception and Performance, 28, 1345-1363.

Swick, D., \& Jovanovic, J. (2002). Anterior cingulate and the Stroop task: Neuropsychological evidence for topographic specificity. Neuropsychologia, 40(8), 1240-1253.

Tzelgov, J., Henik, A., \& Berger, J. (1992). Controlling Stroop effects by manipulating expectations for color words. Memory and Cognition, 20, 727-735.

Ullsperger, M., Bylsma, L. M., \& Botvinick, M. M. (2005). The conflictadaptation effect: It's not just priming. Cognitive, Affective, and Behavioral Neuroscience, 5, 467-472.

Verbruggen, F., Liefooghe, B., Notebaert, W., \& Vandierendonck, A. (2005). Effects of stimulus-stimulus compatibility and stimulusresponse compatibility on response inhibition. Acta Psychologica, 120, 307-326.

Wendt, M., Kluwe, R. H., \& Peters, A. (2006). Sequential modulations of interference evoked by processing task-irrelevant stimulus features. Journal of Experimental Psychology: Human Perception and Performance, 32(3), 644-667.

Received January 3, 2006

Revision received January 1, 2007

Accepted January 18, 2007 